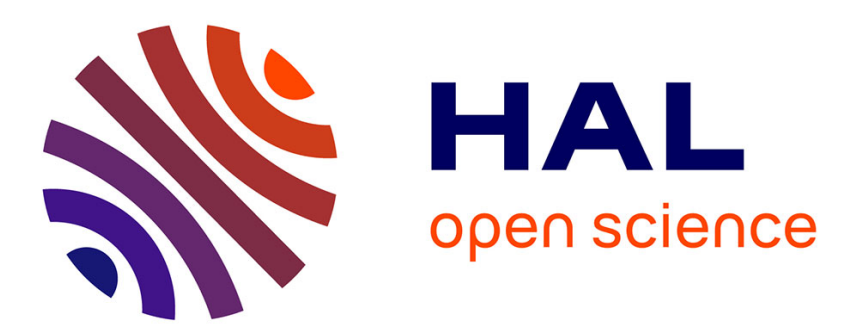

\title{
Simultaneous Drag Reduction and Kinetic Energy Density of the Plane Poiseuille Flow \\ Xuan-Quy Dao, Christophe Collewet
}

\section{To cite this version:}

Xuan-Quy Dao, Christophe Collewet. Simultaneous Drag Reduction and Kinetic Energy Density of the Plane Poiseuille Flow. 6th AIAA Flow Control Conference, Jun 2012, New Orleans, United States. hal-00707173

\section{HAL Id: hal-00707173 \\ https://hal.inria.fr/hal-00707173}

Submitted on 12 Jun 2012

HAL is a multi-disciplinary open access archive for the deposit and dissemination of scientific research documents, whether they are published or not. The documents may come from teaching and research institutions in France or abroad, or from public or private research centers.
L'archive ouverte pluridisciplinaire HAL, est destinée au dépôt et à la diffusion de documents scientifiques de niveau recherche, publiés ou non, émanant des établissements d'enseignement et de recherche français ou étrangers, des laboratoires publics ou privés. 


\title{
Simultaneous Drag Reduction and Kinetic Energy Density of the Plane Poiseuille Flow
}

\author{
Xuan-Quy Dao and Christophe Collewet \\ IRSTEA / INRIA Rennes - Bretagne Atlantique, Fluminance team, Rennes, France.
}

\begin{abstract}
We present in this paper a closed-loop approach to control the 2D plane Poiseuille flow in an unstable state. To do that, we propose to use a vision-based approach as proposed in our paper to estimate the state of the flow ${ }^{1}$. The first step is based on the evaluation of the 2D velocity field from optical flow measurements. These measurements are next used into a visual servo control scheme. However, since we are interested in the simultaneous minimization of two quantities, that is the drag and kinetic energy density of the flow, we propose to design a control law based on an advanced visual servo control approach, the so-called partitioned visual servo control ${ }^{2}$, first proposed in the robotics community. Simulations results validate our control scheme, including the case where noisy measurements are considered. We also compare our approaches with the most relevant ones.
\end{abstract}

\section{Introduction}

Flow control techniques allow to change the current state of a flow to another state or to maintain its current state whatever external disturbances. This is of great economical interest, especially in aeronautics. For instance, Airbus expects in 2020 to decrease by $50 \%$ the CO2 emissions, a large part of this decrease being expected from flow control by diminishing the fuel consumption of their aircrafts through drag reduction ${ }^{3}$.

Passive or active strategies can be considered to control a flow. Passive control consists most often in optimizing shapes or in choosing suitable surfacing such as riblets ${ }^{4}$ for example, the action is thus permanent. Of course, the main drawback of such an approach is its inability to adapt to another situations than the one it has been thought for. In contrast, active control may produce a time varying action. To do that an external energy is required to act on the flow, like techniques based on blowing and suction ${ }^{5}$. This kind of approach can be seen therefore as an optimal problem where one has to apply an optimal control law based on a certain $\operatorname{cost}^{6}$ (minimization of the drag, minimization of the actuators power, etc.). However, very often, open-loop control strategies or even most often forcing strategies ${ }^{7}$ are used. The most important drawbacks of these strategies are that an accurate knowledge of the flow is required leading consequently to high computational costs; in addition, contrary to closed-loop control, these strategies are not robust at all to variations of unmodelled parameters of the system. They can thus painfully cope with real applications.

On the other hand, designing a closed-loop control law requires the use of sensors that have to be at the same time non-intrusive, accurate and adapted to the time and space scales of the phenomenon being controlled. However, non-intrusive sensors are hardly available in the real context of control applications. The most commonly used measurement, obtained from Micro Electro Mechanical Systems (MEMS), is the shear stress at a limited set of measurement points ${ }^{8}$. Thereafter, an observer is required to estimate the state of the flow that will be used in the control law. Indeed, the shear stress based LQG regulator is the standard effective approach for flow control ${ }^{6,8,9}$. In our previous paper ${ }^{1}$ this estimation is obtained from visual measurements. More precisely, the optical flow ${ }^{10}$ is computed and used to build an observer-free closed-loop control law. Our vision-based approach has been revealed to be much more robust than the classical approaches ${ }^{6,8,9}$; it is no more concerned with the well known problem of initialization issue when an observer is used. It has also been shown to be highly robust to measurements noise since a large number of measurements is available. To compare our approach with the classical one we also used in ${ }^{1}$ the LQG control law to control the flow. In this paper, since visual measurements are considered, we aim to fully exploit the capabilities of visual servoing techniques ${ }^{11}$ by designing a more efficient control law. 
The main contribution of this paper is to show that, by fully exploiting the controlled degrees of freedom, a control law can be derived to simultaneously reduce the drag and the kinetic energy density of the flow. To do that we propose to adapt a partitioned visual servo control approach ${ }^{2}$ to the flow control problem. Indeed, this approach has been developed for robotic applications. We will validate this approach on the 2D plane Poiseuille flow. Note that Aamo et al. ${ }^{12}$ has been proposed an interesting approach to cope with this problem, in this work both a drag reduction and a reduction of the kinetic energy density have been obtained. However, tangential actuation has been used. This way to acts has been revealed to lead to lower controllability than wall normal actuation ${ }^{13}$. This is why, the more classical wall normal actuation is preferred in this paper as in in $^{6,9,14-16}$ for example .

This paper is organized as follows: First we present some fundamentals of visual servo control in Section II. Section III presents some basics of the 2D plane Poiseuille controlled flow and the modeling of some physical quantities that will be involved in the control law. The control scheme is detailed in Section IV including the study of the case when the closed loop system is perturbed by measurement noise. Section V is dedicated to simulation results, we compare our approach with the most relevant ones.

\section{Visual servo control}

Visual servo control or visual servoing consists in using the information provided by a vision sensor to control a dynamic system ${ }^{17}$. It is now a well established technique in the robotics community. Indeed, this technique has shown impressive results in numerous complex situations such as underwater, medical and aerial (helicopters, blimps) robotics ${ }^{18}$.

Classically, to achieve a visual servoing task, a set of visual features $\mathbf{s}(t)$ is selected from the image of the scene being observed to control the desired degrees of freedom of the system. A control law is then designed so that this set of visual features $\mathbf{s}(t)$ reaches a desired value $\mathbf{s}^{*}$, corresponding to a desired state of the system, leading to a correct realization of the task. In our case, the visual features will be build from the $2 \mathrm{D}$ velocity field and from the drag as detailed in the next section.

The control principle relies to the regulation to zero of the error vector $\mathbf{e}(t)=\mathbf{s}(t)-\mathbf{s}^{*}$. Its dynamics is given by

$$
\dot{\mathbf{e}}(t)=\frac{\partial \mathbf{e}(t)}{\partial t}+\mathbf{L}_{\mathbf{e}}(t) \mathbf{u}(t)
$$

where $\mathbf{u}(t)$ is the system control inputs, $\mathbf{L}_{\mathbf{e}}(t)$ is the so-called interaction matrix ${ }^{19}$ or the image jacobian that links the time variation of the visual features to the variation of the control signal acting on the system and $\partial \mathbf{e}(t) / \partial t$ expresses the variation of the error vector due to the free motion of the visual features. The interaction matrix plays an essential role. Indeed, the control law is built from its knowledge or from its approximation. It also requires a measurement of the free motion of the visual features.

\section{Modeling issues for the control of the plane Poiseuille flow}

In this section we present the control principle of the plane Poiseuille flow and describe how a reduced linearized model around the steady state can be obtained. The modeling of skin friction drag and the kinetic energy density are next presented.

\section{A. Basics}

Poiseuille flow is a flow that moves between two infinite plates due to a pressure gradient. Fig. 1 illustrates the steady state velocities profile in a streamwise period according to the conceptual model proposed by Joshi et al. ${ }^{8}$. The $x$ axis is associated with the streamwise direction while the $y$ axis is associated to the wall normal direction. The non dimensionalized Navier-Stokes equations (NSE) of this flow are given by

$$
\left\{\begin{array}{l}
\frac{\partial \mathbf{V}}{\partial t}+(\boldsymbol{\nabla} \mathbf{V}) \mathbf{V}=-\boldsymbol{\nabla} P+\frac{1}{R_{e}} \nabla^{2} \mathbf{V} \\
\boldsymbol{\nabla} \cdot \mathbf{V}=0 \\
\mathbf{V}(x, y= \pm 1, t)=\mathbf{0}
\end{array}\right.
$$




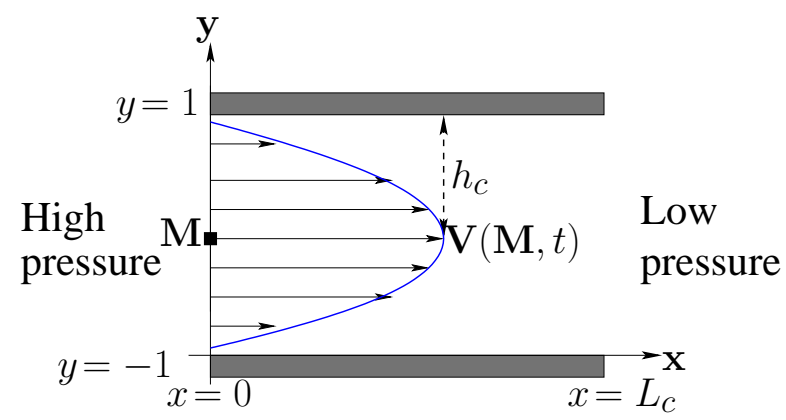

Figure 1. Steady state velocities profile of the 2D plane Poiseuille flow: $h_{c}$ is the channel half height, $L_{c}$ is the flow streamwise period length and $M$ is a point in the flow.

where $P$ is the pressure; $\mathbf{V}$ is the flow velocity; $\mathbf{V}(x, y= \pm 1, t)=\mathbf{0}$ represents the no slip boundary condition; $R_{e}$ is the Reynolds number; $\boldsymbol{\nabla}$ represents the gradient operator and $\nabla^{2}$ the Laplacian.

Since the Poiseuille flow is simple, from (2), it is easy to obtain the analytical solution $\left(V_{b x}, V_{b y}, P_{b}\right)$ in the steady state case, that is when $\frac{d \mathbf{V}}{d t}=\frac{\partial \mathbf{V}}{\partial t}+\mathbf{V} \cdot \nabla \mathbf{V}=\mathbf{0}$ :

$$
\left(V_{b x}, V_{b y}, P_{b}\right)=\left(1-y^{2}, 0,-\frac{2}{R_{e}} x\right) .
$$

The most common way to control this flow is boundary control, it consists in modifying the boundary conditions of the system (2) either only on the lower boundary $y=-1^{8}$, or on both the upper $y=1$ and lower $y=-1$ boundaries $^{9}$.

\section{B. Reduced linearized model of Poiseuille flow}

For a practical implementation of flow control methods, the infinite dimension of a flow requires of a reduced flow model. This section aims at deriving the reduced model of the controlled Poiseuille flow required to derive the control law

Concerning Poiseuille flow, most of the works focus on temporal instabilities caused by a perturbation velocity $\mathbf{V}_{\mathbf{p}}(x, y, t)$. In order to keep permanent such instabilities in the infinite channel when the flow is not controlled, a periodic boundary finite length channel is assumed ${ }^{8}$. That is why the perturbation velocity $\mathbf{V}_{\mathbf{p}}(x, y, t)$ can be expanded in a Fourier series

$$
\mathbf{V}_{\mathbf{p}}(x, y, t)=\sum_{n=-\infty}^{+\infty} V_{p}^{n}(y, t) e^{i \alpha_{n}}
$$

where $\alpha_{n}=n \alpha_{0}$, with $\alpha_{0}=\frac{2 \pi}{L_{c}}$ the fundamental wavenumber and $L_{c}$ is the streamwise period length (see Fig. 1).

The modeling process consists first of all in linearizing the NSE around the steady state solution (3). Then the continuous linearized model of the NSE is reduced by approximation of the perturbation velocity $\mathbf{V}_{\mathbf{p}}(x, y, t)$ at a specifically selected wavenumber $\alpha_{n}$ of the Fourier series (4); and by decomposition of the Fourier series coefficient $V_{p}^{n}(y, t)$ through the evaluation of combinations of Chebychev polynomials $\Phi_{m}$ at Gauss-Lobatto collocation points $y_{k}$ as follows

$$
V_{p}^{n}\left(y_{k}, t\right)=\sum_{m=1}^{M} x_{m}^{n}(t) \Phi_{m}\left(y_{k}\right) \text { with } 1 \leq k \leq M .
$$

where the $x_{m}^{n}(t)$ are the components of the state $\mathbf{x}^{n}(t)$.

Finally, the null boundary conditions of the closed-loop control system is obtained by setting the upper and lower boundaries to the values of the control inputs respectively. All computation done, we obtain the model we are looking for $\left(\mathrm{see}^{9}\right.$ for all the details):

$$
\left\{\begin{array}{l}
\dot{\mathbf{x}}^{n}(t)=\mathbf{A}^{n} \mathbf{x}^{n}(t)+\mathbf{B}^{n} \mathbf{u}(t) \\
\mathbf{x}^{n}(0)=\mathbf{x}^{n}{ }_{0}
\end{array}\right.
$$




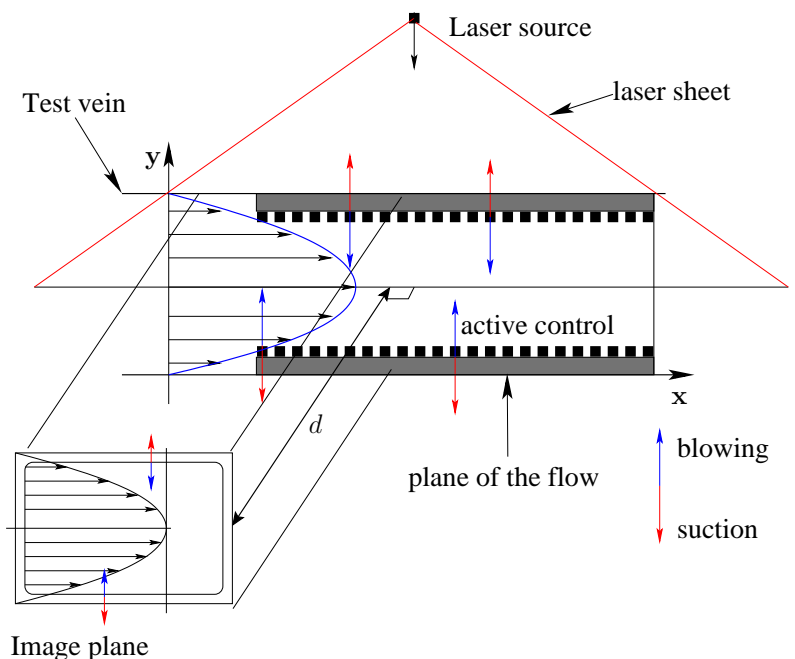

Figure 2. Visualization of the flow using a laser sheet which role is to enlighten the particles seeded in the fluid.

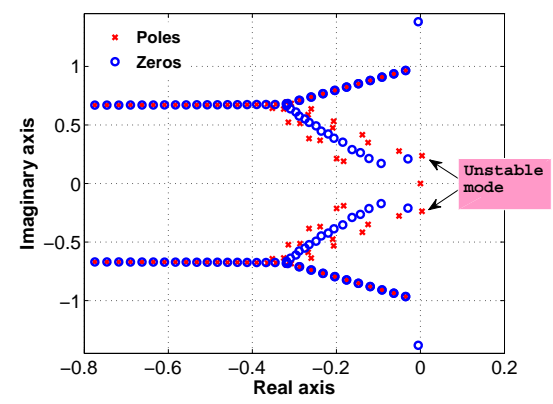

Figure 3. Poles and zeros of the reduced linearized system for $R e=10000$ and $\alpha_{n}=1$.

where $\mathbf{A}^{n}$ is the state matrix, $\mathbf{u}(t)=\left(u_{u}(t), u_{l}(t)\right)$ is the system input (i.e. blowing and suction actions at the channel boundaries), $\mathbf{B}^{n}$ is the input matrix. Recall that in our case ${ }^{1}$ the state vector is directly obtained from the computation of the optical flow through a visualization system as shown in Fig. 2. Let us recall that optical flow is the apparent velocity vector field representing the motion of photometric pattern (pixels luminance) in successive image sequences. Optical flow techniques can be used to estimate instantaneous velocities of a flow from image sequences as detailed in ${ }^{10}$ for example.

In the case of Reynolds number $R e=10000$ and wavenumber $\alpha_{n}=1$, the reduced linearized model of Poiseuille flow (6) is unstable as shown $i^{20}$. In this case the flow is initially in the steady state but in an unstable equilibrium, i.e. a small velocity pertubation value $\mathbf{V}_{p}(x, y, t)$ destabilizes the non-controlled flow. This phenomenon is easy explained through the poles of the matrix $\mathbf{A}^{n}$, a pole has a real part positive (see Fig. 3). We will consider this case in the remainder of this paper. Note also that we will omit now the upperscript $n$ involved in (6) for the sake of clarity.

Note that, even if our main goal is to simultaneously minimize the drag and the kinetic energy density, we have also to ensure the stabilization of the flow, i.e. maintaining the flow in the steady state, whatever the external perturbations.

\section{Modeling of the skin friction drag}

The skin friction drag $d(t)$ due to the perturbation is given by (see e.g. ${ }^{12}$ )

$$
d(t)=-\frac{\partial V_{p x}(x,+1, t)}{\partial y}+\frac{\partial V_{p x}(x,-1, t)}{\partial y}
$$


where $V_{p x}(x,+1, t)$ and $V_{p x}(x,-1, t)$ are the streamwise components of the perturbation velocity $\mathbf{V}_{p}(x, y, t)$ at the walls. Note that $\partial \mathbf{V}_{p x}(x,-1, z, t) / \partial y$ and $\partial \mathbf{V}_{p x}(x,+1, z, t) / \partial y$ are the components of the wall shear stress $\mathbf{s}(t)$ due to the perturbation:

$$
\mathbf{s}(t)=\left[\begin{array}{l}
\frac{\partial V_{p x}(x,+1, z, t)}{\partial y} \\
\frac{\partial V_{p x}(x,-1, z, t)}{\partial y}
\end{array}\right] .
$$

Following the modeling process recalled in section $\mathrm{B}$, it has been shown in ${ }^{9}$ that $\mathbf{s}(t)$ writes simply as a linear function of the state through a matrix $\mathbf{W}$

$$
\mathbf{s}(t)=\mathbf{W} \mathbf{x}(t)
$$

leading consequently from (7) to

$$
d(t)=\mathbf{D}^{\top} \mathbf{x}(t)
$$

with $\mathbf{D}^{\top}=\left[\begin{array}{ll}-1 & +1\end{array}\right] \mathbf{W}$ where $\mathbf{D}^{\top}$ denotes the conjugate transpose of $\mathbf{D}$.

\section{Modeling of the kinetic energy density}

The kinetic energy density of flow perturbation is given by

$$
\varepsilon(t)=\frac{1}{V_{0}} \int_{V_{0}} \frac{\left\|\mathbf{V}_{P}(x, y, t)\right\|^{2}}{2} d V_{0}
$$

where $V_{0}$ is the volume of a period of the domain under consideration. It is possible to obtain a weighting matrix $\mathbf{Q}$ exactly as in ${ }^{21}$ such that the kinetic energy density reduces to

$$
\varepsilon(t)=\mathbf{x}(t)^{\top} \mathbf{Q} \mathbf{x}(t) .
$$

All these physical quantities having been defined, the control law can now be derived.

\section{Control of the 2D plane Poiseuille flow}

As mentioned in the introduction of this paper, our goal is to simultaneously minimize the drag and the kinetic energy density since the flow is in unstable state (see section B). To achieve this goal we propose to use the so-called partitioned visual servo control ${ }^{2}$. This approach has been developed in the robotics domain to solve a very different problem. It consists in decoupling the rotational motions from the translational ones of a robot. To do that, we have first ton compute the interaction matrix $\mathbf{L}_{d}$ of the drag.

\section{A. Computation of the interaction matrix related to the drag}

This computation is however straightforward. Indeed, according to definition of the interaction matrix ${ }^{19}$, we have to express the total time variation of the drag

$$
\dot{d}(t)=\mathbf{L}_{d} \mathbf{u}(t)+\frac{\partial d(t)}{\partial t} .
$$

$\mathbf{L}_{d}$ expresses the variation of the drag due to the actions, while $\partial d(t) / \partial t$ expresses the variation of the drag due to the flow itself.

This computation can be done by derivating (10) with respect to the time and next using (6). We obtain

$$
\left\{\begin{array}{cl}
\mathbf{L}_{d} & =\mathbf{D}^{\top} \mathbf{B} \\
\frac{\partial d(t)}{\partial t} & =\mathbf{D}^{\top} \mathbf{A x}(t) .
\end{array}\right.
$$

Let us introduce the components of the interaction matrix related to the components of the input signal $\mathbf{u}(t)$ (see $(6))$ :

$$
\mathbf{L}_{d}=\left(L_{d u}, L_{d l}\right) .
$$

This expression will be useful in the next section to introduce the partitioned visual servo control. 


\section{B. Design of the control law}

Partitioned visual servo control relies on the partition of the interaction matrix. From (15), the time variation of the drag (13) becomes

$$
\dot{d}(t)=L_{d u} u_{u}(t)+L_{d l} u_{l}(t)+\frac{\partial d(t)}{\partial t} .
$$

Since a decreasing of the drag is desired, we impose a particular behavior for this decreasing. As usually in visual servoing, an exponential decrease is considered

$$
\dot{d}(t)=-\lambda d(t)
$$

where $\lambda$ is a positive gain able to tune the decrease rate. Thereafter, from (16) and (17), we have

$$
-\lambda d(t)=L_{d u} u_{u}(t)+L_{d l} u_{l}(t)+\frac{\partial d(t)}{\partial t} .
$$

Any of the two components of $\mathbf{u}(t)$ can be used to reduce the drag, we arbitrary choose $u_{l}(t)$. We thus have

$$
u_{l}(t)=-\frac{1}{L_{d l}}\left(\lambda d(t)+L_{d u} u_{u}(t)+\frac{\partial d(t)}{\partial t}\right) .
$$

Since the lower boundary control law $u_{l}(t)$ is known if the upper boundary control law $u_{u}(t)$ is known, the next step is to express $u_{u}(t)$.

In order to minimize the kinetic energy density (12), the energy consumption of actuators and to ensure the stabilization of the flow, a LQR control scheme over an infinite time horizon is used by considering the following cost function

$$
J=\int_{0}^{\infty}\left(\mathbf{x}^{\top}(t) \mathbf{Q x}(t)+r u_{u}^{2}(t)\right) \mathrm{d} t .
$$

This can be done by expressing the time variation of the state vector with respect to the control signal $u_{u}$. To do that, we first rewrite (19) from (14) to exhibit the state vector

$$
u_{l}(t)=-\frac{1}{L_{d l}}\left(\left(\lambda \mathbf{D}^{\top}+\mathbf{D}^{\top} \mathbf{A}\right) \mathbf{x}(t)+L_{d u} u_{u}(t)\right)
$$

that we have to plug in (6) leading to

$$
\dot{\mathbf{x}}(t)=\mathbf{A}_{1} \mathbf{x}(t)+\mathbf{B}_{1} u_{u}(t)
$$

where we have introduced the following matrices $\mathbf{A}_{1}=\mathbf{A}-\frac{B_{l}}{L_{d l}}\left(\lambda \mathbf{D}^{\top}+\mathbf{D}^{\top} \mathbf{A}\right)$ and $\mathbf{B}_{1}=\mathbf{B}_{u}-\frac{L_{d u}}{L_{d l}} \mathbf{B}_{l}$ with $\mathbf{B}=\left(\mathbf{B}_{u}, \mathbf{B}_{l}\right)$. Note that $L_{d l}$ is always non null.

Thereafter, it becomes straightforward to compute the LQR gain $\mathbf{k}$ involved in the optimal control $u_{u}=-\mathbf{k}^{\top} \mathbf{x}(t)$ by considering (22) and solving the algebraic Ricatti equation.

Note that here, contrary to the works involved in flow control, we have fully exploited the two controlled degrees of freedom of the 2D plane Poiseuille flow.

The next section is dedicated to the study of the behavior of the closed loop system when measurement noise is considered.

\section{Behavior of the closed loop system in presence of measurement noise}

Of course, when measurement noise occurs, the state estimation $\hat{\mathbf{x}}(t)$ may be very different from the true value $\mathbf{x}(t)$ of the state. In that case, the upper control signal becomes

$$
\hat{u}_{u}=-\mathbf{k}^{\top} \hat{\mathbf{x}}(t) .
$$

The problem is then to verify that $\mathbf{x}(t)$ will still converge towards zero and that the drag is still a decreasing function.

First, we have to express $\hat{\mathbf{x}}(t)$ in function of $\mathbf{x}(t)$. This computation has been done in ${ }^{1}$ in the context of our vision-based approach. In that case, the state estimation writes as

$$
\hat{\mathbf{x}}(t)=\mathbf{x}(t)+\frac{1}{N_{x}} \mathbf{e}_{x}(t)
$$


where $N_{x}$ is the number of pixels of the camera in the streamwise direction of the flow and $\mathbf{e}_{x}(t)$ is related to the measurements noise ( $\mathrm{see}^{1}$ for more details).

Consequently, from (23), (22) becomes

$$
\dot{\mathbf{x}}(t)=\left(\mathbf{A}_{1}-\mathbf{B}_{1} \mathbf{k}^{\top}\right) \mathbf{x}(t)-\mathbf{B}_{1} \mathbf{k} \boldsymbol{\delta}(t)
$$

where the estimation error $\boldsymbol{\delta}(t)=\hat{\mathbf{x}}(t)-\mathbf{x}(t)$ has been introduced. It is clear from (24) that the larger the value of $N_{x}$ the closer $\hat{\mathbf{x}}(t)$ is from $\mathbf{x}(t)$. Indeed, when $N_{x}$ is large enough (it is always the case in practice), the estimation error tends toward 0 , and, consequently the closed loop system (25) writes as

$$
\dot{\mathbf{x}}(t)=\left(\mathbf{A}_{1}-\mathbf{B}_{1} \mathbf{k}^{\top}\right) \mathbf{x}(t)
$$

which is stable.

The other issue concerns the drag. As already mentioned, we have to verify that it is still a decreasing function. To do that, we exam (16) by considering $\hat{u}_{u}, \hat{u}_{l}$ and $\partial \hat{d}(t) / \partial t$. From (21), we have

$$
\hat{u}_{l}(t)=-\frac{1}{L_{d l}}\left(\left(\lambda \mathbf{D}^{\top}+\mathbf{D}^{\top} \mathbf{A}\right) \hat{\mathbf{x}}(t)+L_{d u} \hat{u}_{u}(t)\right)
$$

that writes simply in function of $\mathbf{x}(t)$ by considering (23) and (24)

$$
\hat{u}_{l}(t)=-\frac{1}{L_{d l}}\left(\lambda \mathbf{D}^{\top}+\mathbf{D}^{\top} \mathbf{A}-L_{d u} \mathbf{k}^{\top}\right)\left(\mathbf{x}(t)+\frac{1}{N_{x}} \mathbf{e}_{x}(t)\right) .
$$

The last step is the computation of $\partial \hat{d}(t) / \partial t$, it can be easy done from (14) and (24). All computations done, (16) becomes

$$
\dot{d}(t)=-\lambda d(t)-\frac{\lambda \mathbf{D}^{\top}+\mathbf{D}^{\top} \mathbf{A}}{N_{x}} \mathbf{e}_{x}(t) .
$$

Here again, since $N_{x}$ is a large value, we can consider than the drag follows the desired behavior $\dot{d}(t)=-\lambda d(t)$.

These results will be validated in the next section.

\section{Simulation results}

The simulation results are based on the code provided in $^{13}$. As in $^{6,8,9}$, we consider $R_{e}=10000$ and a channel length $L_{c}=4 \pi$ (see Fig. 1). The scalar $r$ involved in (20) has been set to 2.5 while $\lambda$, involved in the desired behavior of the drag, has been set to 0.5 .

\section{Simultaneous minimization of the drag and the kinetic energy density}

First, as can be seen on Fig. 4, the goal of the control is reached: the control law converges since the input signal tends toward zero (Fig. 4a). Consequently, since a state feedback control law has been used, the state also tends toward zero, that means that the flow stabilizes. Moreover, as expected, an exponential decrease of the drag is obtained (Fig. 4b) as well as a monotonic decrease of the kinetic energy density (Fig. 4c). These results are confirmed on Fig. 5 where the behavior of the velocity field during the servoing is depicted. The flow is first not controlled (Fig. 5a) and becomes thus turbulent due to the presence of a temporal perturbation and because its unstable state. After the application of the input signal, the flow tends towards a laminar behavior (Fig. 5f) but, of course, still in an unstable state.

\section{Comparison with relevant approaches}

We compare now our approach with the most relevant ones, that is ${ }^{8,16,21}$. Indeed, we consider that ${ }^{14,15}$ are similar in mind than ${ }^{16}$. We respectively denote these approaches $P, L Q R_{2}$ and $L Q R_{1}$, we denote ours by $P V S$ (standing for partitioned visual servoing). Let us briefly describe these works. $\mathrm{In}^{8}$, a proportional output feedback control is used, the output is the wall shear stress. Therefore, vanishing the shear stress also vanishes the drag. In this approach the kinetic energy density is not considered. $\mathrm{In}^{21}$ the kinetic energy density is minimized by using a LQR approach. The matrix $\mathbf{Q}$ involved in (12) is used in the cost function that we have to minimize. Note that is an indirect way to minimize the drag since the drag vanishes when the 


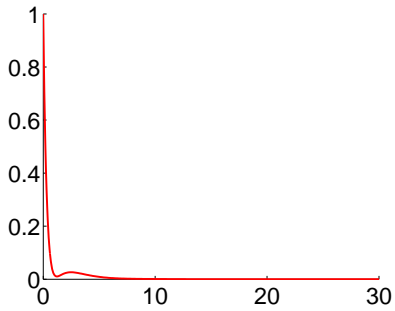

(a)

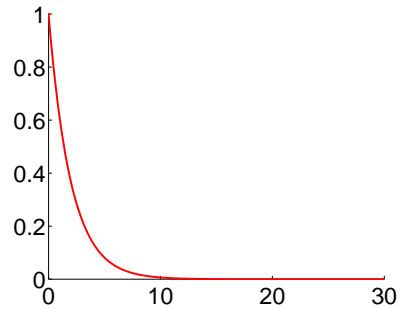

(b)

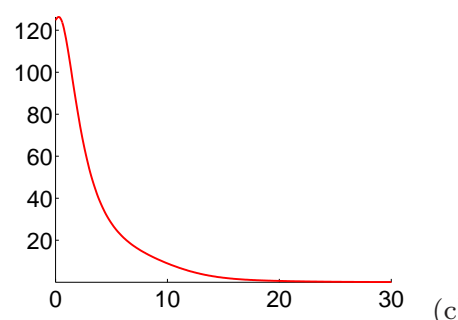

(c)

Figure 4. Convergence of the control law ( $x$ axis in second). (a) Norm of the input signal u(t) vs time; (b) drag vs time; (c) kinetic energy density vs time.
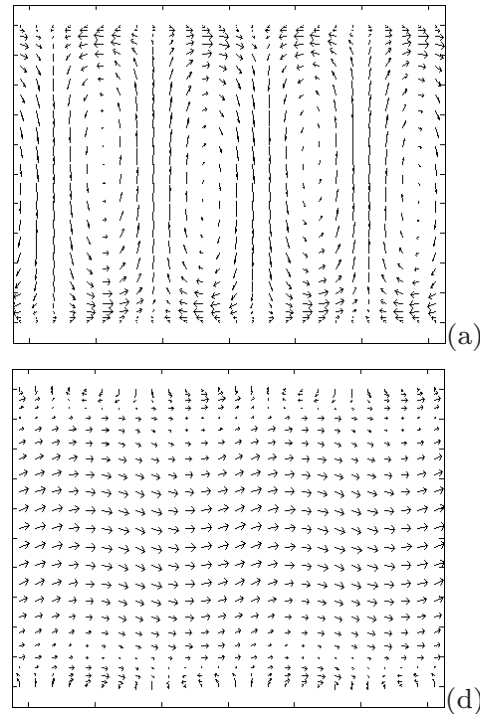
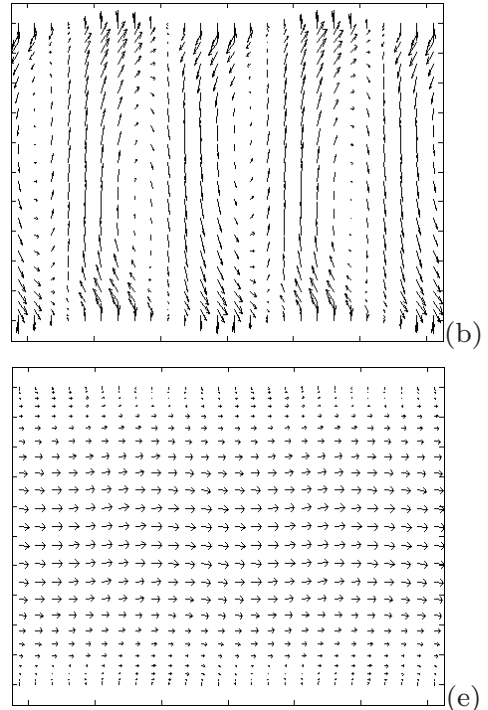
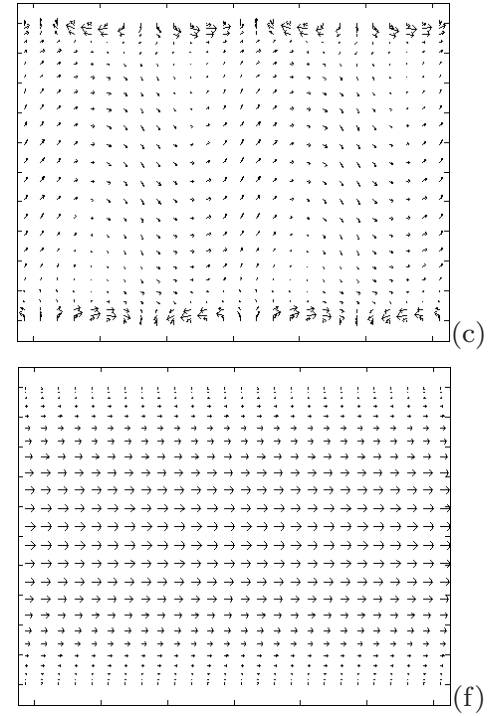

Figure 5. Velocity field vs time. (a) due to an initial perturbation the flow becomes turbulent; (b) first iteration of the control law; (c) and (d) intermediate state of the controlled flow; (e) the flow becomes laminar; (f) the control law converged, the flow is laminar in an unstable state.

kinetic energy density does. Finally, in ${ }^{16}$, the wall shear stress is minimized also through a LQR approach. Note that we cannot exactly compared our approach with these works since none of them simultaneously minimizes the drag and the kinetic energy density.

The results are depicted on Fig. 6. More precisely, Fig. 6a depicts the behavior of the drag versus time while Fig. 6b depicts the behavior of the kinetic energy density versus time. As expected, our approach provides better results than the other approaches. A nice decreasing of the drag and the kinetic energy density are observed. The $L Q R_{1}$ approach leads also to a nice minimization of the kinetic energy density but the (indirect) minimization of the drag is not satisfactory. Both methods based on the minimization of the shear stress $\left(P\right.$ and $\left.L Q R_{2}\right)$ lead to a worse behavior than $P V S$ and $L Q R_{1}$. Note also that, except for our approach, the decreasing of the drag towards zero is very low.

However, the input signal provides both by the $P$ and $L Q R_{2}$ methods are very low in contrast to the $P V S$ and $L Q R_{1}$ approaches (see Fig. 7). Therefore, by tuning the matrix $\mathbf{R}$ involved in the $L Q R_{2}$ approach or the gain for the $P$ approach better results could be expected. Unfortunately, it is not the case as proved by the results depicted on Fig. 8. In particular, oscillations occur in the kinetic energy density (Fig. 8b).

\section{Influence of the parameter $\lambda$ on the behavior of the flow}

Fig. 9 describes the behavior of the system for different values of $\lambda$. More precisely, Fig. 9a depicts the behavior of the drag, as expected an exponential decrease is obtained. Fig. 9b represents the behavior of the kinetic energy density for various choices of $\lambda$, as can be seen the kinetic energy density does not depend, in practice, on this value. That means that the way the drag is reduced does not influence the way the kinetic 


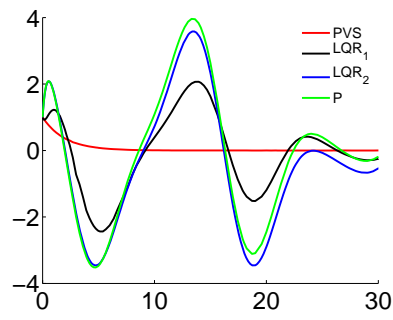

(a)

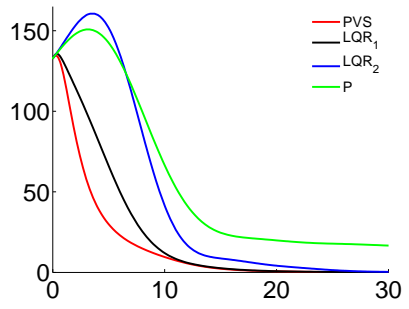

Figure 6. Comparison of the different approaches ( $x$ axis in second). (a) drag vs time; (b) kinetic energy density vs time.

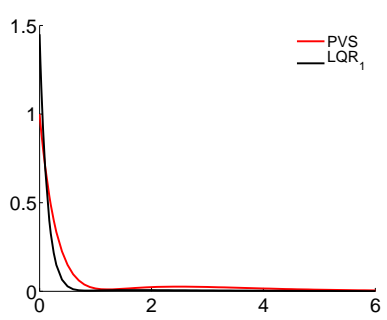

(a)

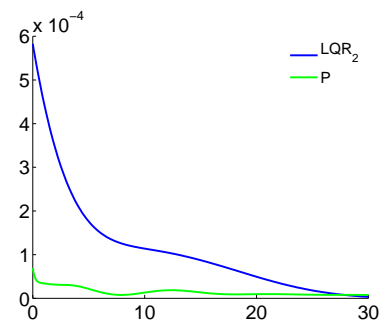

(b)

Figure 7. Comparison of the different approaches ( $x$ axis in second). Norm of the input signal u $(t)$ vs time.

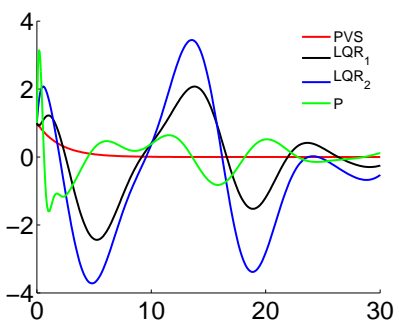

(a)

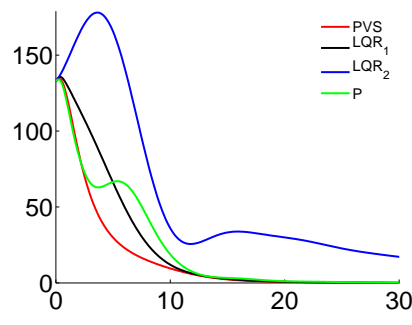

(b)

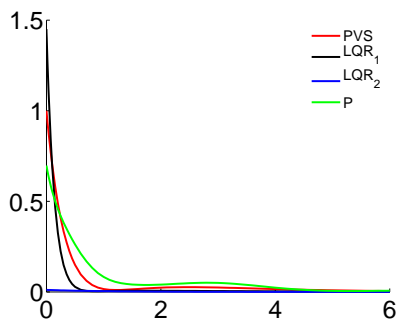

(c)

Figure 8. Comparison of the different approaches ( $x$ axis in second). (a) drag vs time; (b) kinetic energy density vs time (c) Norm of the input signal $\mathbf{u}(t)$ vs time.

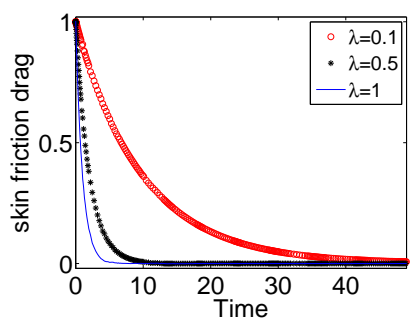

(a)

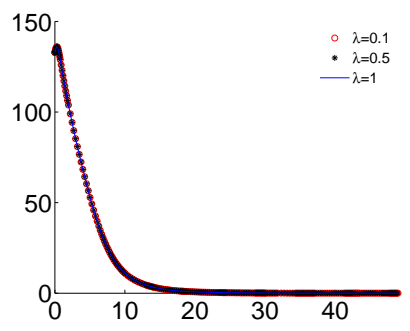

(b)

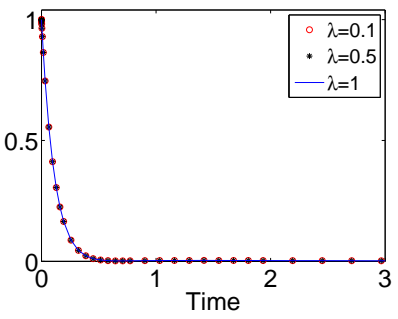

Figure 9. Behavior of the system for different values of $\lambda$ ( $x$ axis in second). (a) drag vs time; (b) kinetic energy density vs time (c) Norm of the input signal $\mathbf{u}(t)$ vs time. 
energy density is reduced. Finally, Fig. 9c depicts the behavior of the control law, it also does not depend in practice on the gain $\lambda$.

\section{Influence of noisy measurements on the behavior of the flow}

The next simulation concerns the behavior of the system when noisy measurement are considered. The results are depicted on Fig. 10, with or without noise. Respectively the drag, the kinetic energy density and the norm of the input signal are represented on Fig. 10a, Fig. 10b and Fig. 10c. A Gaussian noise with a standard deviation of 0.3 has been added to the velocity fields. The number of pixels $N_{x}$ has been set to 2048. As can be seen, despite this very low value, very good results are obtained. In particular, the convergence of the control law is ensured, as well as the decrease of the drag. Note that, contrary to our vision-based estimation scheme, all the works previously described are very sensitive to measurement noise. The control law may be diverged (see ${ }^{1}$ for more details).

\section{Conclusion}

In this paper we have shown the benefit of using an advanced visual servo control approach for the simultaneous minimization of the drag and the kinetic energy density. Our approach clearly outperforms the previous works in this domain even if a rigorous comparison is not possible since none of these works are able to simultaneously minimize both these quantities. Concerning our approach, we have shown that in practice, the way the drag is reduced does not influence the way the kinetic energy density is reduced. In addition, we have proved that our approach is very robust against measurement noise. This nice propriety is due to the large amount of flow velocities available through the computation of the optical flow. In contrast, the existing methods are sensitive to noise since the state estimation is based on shear stress measurements on only few points.

\section{References}

\footnotetext{
${ }^{1}$ Tatsambon Fomena, R. and Collewet, C., "Fluid Flow Control: a Vision-Based Approach," International Journal of Flow Control, Vol. 3, No. 2+3, September 2011, pp. 133-169.

${ }^{2}$ Corke, P. and Hutchinson, S., "A new partitioned approach to image-based visual servo control," IEEE Trans on Robotics and Automation, Vol. 17, No. 4, August 2001, pp. 507-515.

${ }^{3}$ Flaig, A., "Eco-efficient by design," Challenges for aerodynamics engineers for future aircraft design, 8th World Congress on Computational Mechanics, Venice, Italy, 2008.

${ }^{4}$ Choi, H., Moin, P., and Kim, J., "Direct numerical simulation of turbulent flow over riblets," Journal of Fluid Mechanics, Vol. 255, 1993, pp. 503-539.

${ }^{5}$ Wygnanski, I., "Boundary layer flow control by periodic addition of momentum," 4th AIAA Shear Flow Control Conference, USA, June 29-July 2, 1997.

${ }^{6}$ Bewley, T. and Liu, S., "Optimal and robust control and estimation of linear paths to transition," Journal of Fluid Mechanics, Vol. 365, 1998, pp. 305-349.

${ }^{7}$ Protas, B. and Wesfreid, J., "Drag force in the open-loop control of the cylinder wake in the laminar regime," Physics of Fluids, Vol. 14, No. 2, February 2002, pp. 810-826.

${ }^{8}$ Joshi, S., Speyer, J., and Kim, J., "A system theory approach to the feedback stabilization of infinitesimal and finite amplitude disturbances in plane Poiseuille flow," Journal of Fluid Mechanics, Vol. 332, 1997, pp. 157-184.

${ }^{9}$ McKernan, J., Papadakis, G., and Whidborne, J., "A Linear State-Space Representation of Plane Poiseuille Flow for Control Design- A tutorial," International Journal of Modelling, Identification and Control, Vol. 1, No. 4, 2006, pp. $272-280$.

${ }^{10}$ Heitz, D., Mémin, E., and Schnörr, C., "Variational fluid flow measurements from image sequences: synopsis and perspectives," Exp. Fluids, Vol. 48, No. 3, 2010, pp. 369-393.

${ }^{11}$ Chaumette, F. and Hutchinson, S., "Visual Servo Control, Part II: Advanced Approaches," IEEE Robotics and Automation Magazine, Vol. 14, No. 1, March 2007, pp. 109-118.

${ }^{12}$ Aamo, O., Krstic, M., and Bewley, T., "Control of mixing by boundary feedback in 2D channel flow," Automatica, , No. 39, 2003, pp. 1597-1606.

${ }^{13}$ McKernan, J., Control of plane Poiseuille flow: a theoretical and computational investigation, Ph.D. thesis, Cranfield University, 2006.

${ }^{14}$ Joshi, S., Speyer, J., and Kim, J., "Finite dimensional optimal control of Poiseuille flow," Journal of Guidance, Control, and Dynamics, Vol. 22, No. 2, 1999, pp. 340-348.

${ }^{15}$ Lee, K. H., Cortelezzi, L., Kim, J., and Speyer, J. L., "Application of Robust Reduced-Order Controller to Turbulent Flows for Drag Reduction," Physics of Fluids, Vol. 13, No. 5, 2001, pp. 1321-1330.

${ }^{16}$ Cortelezzi, L., Lee, K., Kim, J., and Speyer, J., "Skin-friction Drag Reduction Via Robust Reduced-order Linear Feedback Control," International Journal of Computational Fluid Dynamics, Vol. 11, 1998, pp. 79-92.
} 

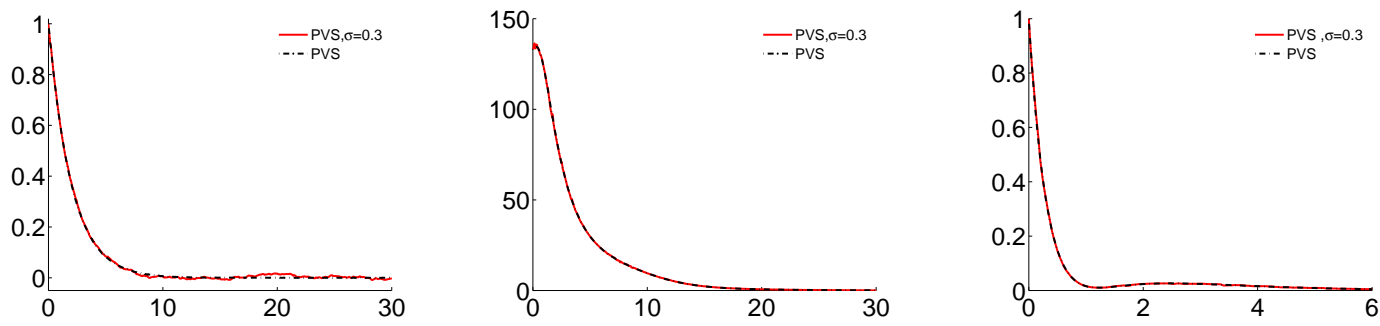

(a)

Figure 10. Behavior when measurement noise is considered ( $x$ axis in second). (a) drag vs time; (b) kinetic energy density vs time; (c) Norm of the input signal $\mathbf{u}(t)$ vs time.

\footnotetext{
${ }^{17}$ Chaumette, F. and Hutchinson, S., "Visual Servo Control, Part I: Basic Approaches," IEEE Robotics and Automation Magazine, Vol. 13, No. 4, December 2006, pp. 82-90.

${ }^{18}$ Bonin-Font, F., Ortiz, A., and Oliver, G., "Visual navigation for mobile robots: a survey," Journal of Intelligent 8 Robotic Systems, Vol. 53, No. 3, 2008, pp. 263-296.

${ }^{19}$ Espiau, B., Chaumette, F., and Rives, P., "A new approach to visual servoing in robotics," IEEE Trans. on Robotics and Automation, Vol. 8, No. 3, June 1992, pp. 313-326.

${ }^{20}$ Orszag, S., "Accurate Solution of the Orr-Sommerfeld Stability Equation," Journal of Fluid Mechanics, Vol. 50, No. 4, 1971, pp. 689-703.

${ }^{21}$ McKernan, J., Whidborne, J., and Papadakis, G., "Linear quadratic control of plane Poiseuille flow - the transient behaviour," International Journal of Control, Vol. 80, No. 12, December 2007, pp. 1912-1930.
} 